\title{
Cut elimination for first order Gödel logic by hyperclause resolution
}

\author{
Matthias Baaz ${ }^{\star}$ Agata Ciabattoni ${ }^{\star \star}$ Christian G. Fermüller ${ }^{\star \star \star}$ \\ Technische Universität Wien, Austria
}

\begin{abstract}
Efficient, automated elimination of cuts is a prerequisite for proof analysis. The method CERES, based on Skolemization and resolution has been successfully developed for classical logic for this purpose. We generalize this method to Gödel logic, an important intermediate logic, which is also one of the main formalizations of fuzzy logic.
\end{abstract}

\section{Introduction}

In recent years an efficient method for the automated elimination of cuts from classical first order sequent proofs has been developed [7,9]. This method, called CERES ${ }^{1}$ is based on the resolution calculus and has been successfully employed for the in depth analysis of proofs in number theory (e.g., [5]). It is moreover also of theoretical interest due to its global nature and other essential differences, compared to the traditional, local Gentzen- and Schütte-Tait-style cut elimination methods $[18,20]$. Of course, effective cut elimination is not only useful for classical logic. It is a precondition for non-trivial proof analysis in any logic. In [8] Baaz and Leitsch have extended CERES to a wide class of finite-valued logics. Considering the intended applications, intuitionistic logic and intermediate logics, i.e., logics over the standard language that are stronger than intuitionistic logic, but weaker than classical logic, are even more important targets for similar extensions. However, there are a number formidable obstacles to a straightforward generalization of CERES to this realm of logics:

- It is unclear whether and how classical resolution can be generalized, for the intended purpose, to intermediate logics.

- Gentzen's sequent format is too restrictive to obtain appropriate analytic calculi for many important intermediate logics.

- Skolemization, or rather the inverse de-Skolemization of proofs - an essential prerequisite for CERES - is not possible in general.

Here we single out a prominent intermediate logic, namely Gödel logic G (also called Dummett's LC or Gödel-Dummett logic), which is also one of the main formalizations of fuzzy logic (see, e.g., [13]) and therefore sometimes called intuitionistic fuzzy

\footnotetext{
^ Partially supported by FWF (Austrian Science Foundation) P19875.

$\star \star$ Partially supported by FWF Project P18731.

$\star \star \star$ Partially supported by ESF/FWF Project I143-G15.

${ }^{1}$ CERES stands for Cut Elimination by RESolution.
} 
logic [22]. We show that essential features of CERES can be adapted to the calculus HG $[1,11]$ for $\mathbf{G}$ that uses hypersequents, a generalization of Gentzen's sequents to multisets of sequents. This adaption is far from trivial and, among other novel features, entails a new concept of 'resolution': hyperclause resolution, which combines most general unification and cuts on atomic hypersequents. It also provides clues to a better understanding of resolution based cut elimination for sequent and hypersequent calculi, in general.

Due to the incorrectness of general de-Skolemization we will deal with HG-proofs with (arbitrary cut-formulas, but) end-hypersequents that contain either only weak quantifier occurrences or only prenex formulas. For the latter case we show that the corresponding class of proofs admits de-Skolemization.

Our results can also be seen as a first step towards automatizing cut elimination and proof analysis for intuitionistic and other intermediate logics.

\section{First order Gödel logic and hypersequents}

First-order Gödel logic $\mathbf{G}$ is one of the most important intermediate logics. It can be characterized semantically by the class of all rooted linearly ordered Kripke models with constant domains, see e.g. [12]. Syntactically, $\mathbf{G}$ arises from intuitionistic logic by adding the axiom of linearity $(A \supset B) \vee(B \supset A)$ and the quantifier shifting axiom $\forall x(A(x) \vee C) \supset[(\forall x A(x)) \vee C]$, where the $x$ does not occur free in $C$.

The importance of the logic is also indicated by the fact that it can alternatively be seen as a fuzzy logic, i.e., as an infinite-valued logic with the real unit interval $[0,1]$ as set of truth values [22,13]; but also as a temporal logic [10].

Hypersequent calculi [2] are simple and natural generalizations of Gentzen's sequent calculi. In our context, a hypersequent is a multiset of single-conclusioned ('intuitionistic') sequents, called components, written as

$$
\Gamma_{1} \Rightarrow \Delta_{1}|\cdots| \Gamma_{n} \Rightarrow \Delta_{n}
$$

where, for $i \in\{1, \ldots, n\}, \Gamma_{i}$ is a multiset of formulas, and $\Delta_{i}$ is either empty or a single formula. The intended interpretation of the symbol ' $\mid$ ' is disjunction at the meta-level.

A hypersequent calculus for propositional Gödel logic has been introduced by Avron [1,2] and extended to first-order in [11]. The logical rules and internal structural rules of this calculus are essentially the same as those in Gentzen's sequent calculus $\mathrm{LJ}$ for intuitionistic logic; the only difference being the presence of contexts $\mathscr{H}$ representing (possibly empty) side hypersequents. In addition we have external contraction and weakening, and the so-called communication rule. We present an equivalent version $\mathrm{HG}$ of the calculi in $[1,11]$ with multiplicative logical rules (see, e.g., [21] for this terminology).

Axioms: $\perp \Rightarrow, \quad A \Rightarrow A$, for atomic $^{2}$ formulas $A$.

In the following rules, $\Delta$ is either empty or a single formula.

\section{Internal structural rules:}

$$
\frac{\mathscr{H} \mid \Gamma \Rightarrow \Delta}{\mathscr{H} \mid A, \Gamma \Rightarrow \Delta}(i w-l) \quad \frac{\mathscr{H} \mid \Gamma \Rightarrow}{\mathscr{H} \mid \Gamma \Rightarrow A}(i w-r) \quad \frac{\mathscr{H} \mid A, A, \Gamma \Rightarrow \Delta}{\mathscr{H} \mid A, \Gamma \Rightarrow \Delta}(\text { ic-l })
$$

\footnotetext{
2 The restriction to atomic axioms is useful, but does not imply any loss of generality.
} 


\section{External structural rules:}

$$
\frac{\mathscr{H}}{\mathscr{H} \mid \Gamma \Rightarrow \Delta}(\text { ew }) \quad \frac{\mathscr{H}|\Gamma \Rightarrow \Delta| \Gamma \Rightarrow \Delta}{\mathscr{H} \mid \Gamma \Rightarrow \Delta}(\text { ec })
$$

\section{Logical rules:}

$$
\begin{array}{cc}
\frac{\mathscr{H}\left|A_{1}, \Gamma_{1} \Rightarrow \Delta \quad \mathscr{H}^{\prime}\right| A_{2}, \Gamma_{2} \Rightarrow \Delta}{\mathscr{H}\left|\mathscr{H}^{\prime}\right| A_{1} \vee A_{2}, \Gamma_{1}, \Gamma_{2} \Rightarrow \Delta}(\vee-l) & \frac{\mathscr{H} \mid \Gamma \Rightarrow A_{i}}{\mathscr{H} \mid \Gamma \Rightarrow A_{1} \vee A_{2}}\left(\vee_{i}-r\right)_{i \in\{1,2\}} \\
\frac{\mathscr{H} \mid A_{i}, \Gamma \Rightarrow \Delta}{\mathscr{H} \mid A_{1} \wedge A_{2}, \Gamma \Rightarrow \Delta}\left(\wedge_{i}-l\right)_{i \in\{1,2\}} & \frac{\mathscr{H}\left|\Gamma_{1} \Rightarrow A \quad \mathscr{H}^{\prime}\right| \Gamma_{2} \Rightarrow B}{\mathscr{H}\left|\mathscr{H}^{\prime}\right| \Gamma_{1}, \Gamma_{2} \Rightarrow A \wedge B}(\wedge-r) \\
\frac{\mathscr{H}\left|\Gamma_{1} \Rightarrow A \quad \mathscr{H}^{\prime}\right| B, \Gamma_{2} \Rightarrow \Delta}{\mathscr{H}\left|\mathscr{H}^{\prime}\right| A \supset B, \Gamma_{1}, \Gamma_{2} \Rightarrow \Delta}(\supset-l) & \frac{\mathscr{H} \mid A, \Gamma \Rightarrow B}{\mathscr{H} \mid \Gamma \Rightarrow A \supset B}(\supset-r)
\end{array}
$$

In the following quantifier rules $t$ denotes an arbitrary term, and $y$ denotes an eigenvariable, i.e., $y$ does not occur in the lower hypersequent:

$$
\begin{array}{cc}
\frac{\mathscr{H} \mid A(t), \Gamma \Rightarrow \Delta}{\mathscr{H} \mid(\forall x) A(x), \Gamma \Rightarrow \Delta}(\forall-l) & \frac{\mathscr{H} \mid \Gamma \Rightarrow A(y)}{\mathscr{H} \mid \Gamma \Rightarrow(\forall x) A(x)}(\forall-r) \\
\frac{\mathscr{H} \mid A(y), \Gamma \Rightarrow \Delta}{\mathscr{H} \mid(\exists x) A(x), \Gamma \Rightarrow \Delta}(\exists-l) & \frac{\mathscr{H} \mid \Gamma \Rightarrow A(t)}{\mathscr{H} \mid \Gamma \Rightarrow(\exists x) A(x)}(\exists-r)
\end{array}
$$

Like in [21] we call the exhibited formula in the lower hypersequent of each of these rules the main formula, and the corresponding subformulas exhibited in the upper hypersequents the active formulas of the inference.

The following communication rule of $\mathrm{HG}$ is specific to logic $\mathbf{G}$ :

$$
\frac{\mathscr{H}\left|\Gamma_{1}, \Gamma_{2} \Rightarrow \Delta_{1} \quad \mathscr{H}^{\prime}\right| \Gamma_{1}, \Gamma_{2} \Rightarrow \Delta_{2}}{\mathscr{H}\left|\mathscr{H}^{\prime}\right| \Gamma_{1} \Rightarrow \Delta_{1} \mid \Gamma_{2} \Rightarrow \Delta_{2}}(\mathrm{com})
$$

This version of the communication is equivalent to the one introduced in [1] (see [2]). Finally we have cut, where $A$ is called cut-formula of the inference:

$$
\frac{\mathscr{H}\left|\Gamma_{1} \Rightarrow A \quad \mathscr{H}^{\prime}\right| A, \Gamma_{2} \Rightarrow \Delta}{\mathscr{H}\left|\mathscr{H}^{\prime}\right| \Gamma_{1}, \Gamma_{2} \Rightarrow \Delta} \text { (cut) }
$$

If $A$ is atomic we speak of an atomic cut.

Remark. Note the absence of negation from our calculus: $\neg A$ is just an abbreviation of $A \supset \perp$. (See, e.g., [21] for similar sytems for intuitionistic logic.)

Communication allows us to derive the following additional 'distribution rule' which we will use in Section 6:

$$
\frac{\mathscr{H} \mid \Gamma \Rightarrow A \vee B}{\mathscr{H}|\Gamma \Rightarrow A| \Gamma \Rightarrow B} \text { (distr) }
$$

A derivation $\rho$ using the rules of HG is viewed as an upward rooted tree. The root of $\rho$ is called its end-hypersequent, which we will denote by $\mathscr{H}_{\rho}$. The leaf nodes are called initial hypersequents. A proof $\sigma$ of a hypersequent $\mathscr{H}$ is a derivation with $\mathscr{H}_{\sigma}=\mathscr{H}$, where all initial hypersequents are axioms.

The ancestors of a formula occurrence in a derivation are traced upwards to the initial hypersequents in the obvious way. I.e., active formulas are immediate ancestors of the main formula of an inference. The other formula occurrences in the premises 
(i.e., upper hypersequents) are immediate ancestors of the corresponding formula occurrences in the lower hypersequent. (This includes also internal and external contraction: here, a formula in the lower hypersequent may have two corresponding occurrences, i.e. immediate ancestors, in the premises.) The ancestor relation is the transitive closure of immediate ancestorship.

The sub-hypersequent consisting of all ancestors of cut-formulas of an hypersequent $\mathscr{H}$ in a derivation is called the cut-relevant part of $\mathscr{H}$. The complementary sub-hypersequent of $\mathscr{H}$ consisting of all formula occurrences that are not ancestors of cut-formulas is the cut-irrelevant part of $\mathscr{H}$. An inference is called cut-relevant if its main formula is an ancestor of a cut-formula, and is called cut-irrelevant otherwise.

The hypersequent $\Gamma_{1} \Rightarrow \Delta_{1}|\ldots| \Gamma_{n} \Rightarrow \Delta_{n}$ is called valid if its translation $\bigvee_{1 \leq i \leq n}\left(\bigwedge_{A \in \Gamma_{i}} A \supset\left[\Delta_{i}\right]\right)$ is valid in $\mathbf{G}$, where $\left[\Delta_{i}\right]$ is $\perp$ if $\Delta_{i}$ is empty, and the indicated implications collapse to $\Delta_{i}$ whenever $\Gamma_{i}$ is empty. A set of hypersequents is called unsatisfiable if their translations entail $\perp$ in $\mathbf{G}$. (Different but equivalent ways of defining validity and entailment in $\mathbf{G}$ have been indicated at the beginning of this section.)

Theorem 1 ([11,3]). A hypersequent $\mathscr{H}$ is provable in $\mathrm{HG}$ without cuts iff $\mathscr{H}$ is valid.

Remark. It might surprise the reader that we rely on the cut-free completeness of HG in a paper dealing with cut elimination. However, this just emphasizes the fact that we are interested in a particular transformation of proofs with cuts (i.e., 'lemmas') into cut-free proofs, that is adequate for automatization and proof analysis (compare $[9,5]$ ).

\section{Overview of hyperCERES}

Before presenting the details of our transformation of appropriate HG-proofs into cutfree proofs, which we call hyperCERES, we will assist the orientation of the reader and describe the overall procedure on a more abstract level using keywords that will be explained in the following sections. ${ }^{3}$

The end-hypersequent $\mathscr{H}_{\sigma}$ of the HG-proof $\sigma$ that forms the input of hyperCERES can be of two forms: either it contains only weak quantifier occurrences or it consists of prenex formulas only. ${ }^{4}$ In the latter case we have to Skolemize the proof first (step 1) and de-Skolemize it after cut elimination (step 7):

1. if necessary, construct a Skolemized form $\hat{\sigma}$ of $\sigma$, otherwise $\hat{\sigma}=\sigma$ (Section 4)

2. compute a characteristic set of pairs $\left\{\left\langle R_{1}(\hat{\sigma}), D_{1}\right\rangle, \ldots\left\langle R_{n}(\hat{\sigma}), D_{n}\right\rangle\right\}$, where $\Sigma_{d}(\hat{\sigma})=\left\{D_{1}, \ldots, D_{n}\right\}$ is the characteristic set of d-hyperclauses - coding the cut formulas of $\hat{\sigma}$ - and each reduced proof $R_{i}(\hat{\sigma})$ is a cut-free proof of a cut-irrelevant sub-hypersequent of $\mathscr{H}_{\hat{\sigma}}$ augmented by $D_{i}$ (Section 5$)$

3. translate $\Sigma_{d}(\hat{\sigma})$ into an equivalent set of hyperclauses $\Sigma(\hat{\sigma})$ and construct a (hyperclause) resolution refutation $\gamma$ of $\Sigma(\hat{\sigma})$ (Section 6 )

4. compute a ground instantiation $\gamma^{\prime}$ of $\gamma$ using a ground substitution $\theta$ (Section 6)

\footnotetext{
${ }^{3}$ Due to space constraints we have to refer the reader to [9] for a presentation of CERES.

${ }^{4}$ While in classical logic all formulas can be translated into equivalent prenex formulas, this does not hold for $\mathbf{G}$.
} 
5. apply $\theta$ to the reduced proofs $R_{1}(\hat{\sigma}), \ldots, R_{n}(\hat{\sigma})$, and assemble them into a single proof $\gamma^{\prime}[\hat{\sigma}]$ using the atomic cuts and contractions that come from $\gamma$ (Section 7)

6. eliminate the atomic cuts in $\gamma^{\prime}[\hat{\sigma}]$ in the usual way ${ }^{5}$

7. if necessary, de-Skolemize the proof $\gamma^{\prime}[\hat{\sigma}]$ and apply final contractions and weakenings to obtain a cut-free proof of $\mathscr{H}_{\sigma}$ (Section 4)

It is well known (see, e.g., $[19,17])$ that there is no elementary bound on the size of shortest cut-free proofs relative to the size of proofs with cuts of the same end(hyper)sequent. While the non-elementary upper bound on the complexity of cut elimination obviously also applies to hyperCERES it should be pointed out that the global (hyperclause) resolution based method presented here is considerably faster in general, and never essentially slower, than traditional Gentzen- or Schütte-Tait-style cut elimination procedures $[1,3]$. Moreover, the reliance on most general unification and atomic cuts, i.e., on resolution for the computational kernel of the procedure implies that hyperCERES is a potentially essential ingredient of (semi-)automated analysis of appropriately formalized proofs.

\section{Skolemization and de-Skolemization}

Like in the original CERES-method [7,9], step 5 of hyperCERES is sound only if end(hyper)sequents do not contain strong quantifier occurrences. The reason for this is that, in general, the eigenvariable condition might be violated when the reduced proofs (constructed in step 2) are combined with the resolution refutation (constructed in step 3) to replace the original cuts with atomic cuts. Consequently, like in CERES, we first Skolemize the proof; i.e., we replace all strong quantifier occurrences with appropriate Skolem terms. (Obviously this is necessary only if there are strong quantifier occurrences at all.) While this transformation is always sound (in fact also for LJ-proofs), the inverse de-Skolemization, i.e., the re-introduction of strong quantifier occurrences according to the information coded in the Skolem terms, is unsound in general. ${ }^{6}$ However, as we will show below, de-Skolemization is possible for HG-proofs of prenex hypersequents (step 7).

By a prenex hypersequent we mean a hypersequent in which all formulas are in prenex form, i.e., all formulas begin with a (possibly empty) prefix of quantifier occurrences, followed by a quantifier-free formula. If $\Gamma \Rightarrow \Delta$ is a component of a prenex hypersequent, then all existential quantifiers occurring in $\Gamma$ and all universal quantifiers occurring in $\Delta$ are called strong. The other quantifier occurrences are called weak.

The Skolemization $\mathscr{H}^{S}$ of a prenex hypersequent $\mathscr{H}$ is obtained as follows. In every component $\Gamma \Rightarrow \Delta$ of $\mathscr{H}$, delete each strong quantifier occurrence $\mathrm{Q} x$ and replace all corresponding occurrences of $x$ by the Skolem term $f(\bar{y})$, where $f$ is a new function symbol and $\bar{y}$ are the variables of the weak quantifier occurrences in the scope of which $\mathrm{Q} x$ occurs. (If $\mathrm{Q} x$ is not the scope of any weak quantifier then $f$ is a constant symbol.)

Given an HG-proof $\sigma$ of $\mathscr{H}$ its Skolemization $\hat{\sigma}$ is constructed in stages:

\footnotetext{
${ }^{5}$ As is known, atomic cuts in HG-proofs can be moved upwards to the axioms, where they become redundant (see, e.g., $[3,1]$ ).

${ }^{6}$ E.g. $\forall x(A(x) \vee B) \Rightarrow A(c) \vee B$ is provable in $L J$ while its de-Skolemized version $\forall x(A(x) \vee B) \Rightarrow$ $\forall x A(x) \vee B$ is not.
} 
1. Replace the end-hypersequent $\mathscr{H}$ of $\sigma$ by $\mathscr{H}^{S}$. Recall that this means that every occurrence of a strongly quantified variable $x$ in $\mathscr{H}$ is replaced by a corresponding Skolem term $f(\bar{y})$.

2. Trace the indicated occurrences of $x$ and of the eigenvariable $y$ corresponding to its introduction throughout $\sigma$ and replace all these occurrences by $f(\bar{y})$, too.

3 . Delete the (now) spurious strong quantifiers and remove the corresponding inferences that introduce these quantifiers in $\sigma$.

4. For any inference in $\sigma$ introducing a weakly quantified variable $y$ by replacing $A(t)$ with QyA(y), replace all corresponding occurrences of $y$ in Skolem terms $f(\bar{y})$ by $t$.

It is straightforward to check that $\hat{\sigma}$ is an HG-proof of $\mathscr{H}^{S}$. (Note that strong quantifier occurrences in ancestors of cut formulas remain untouched by our Skolemization.)

It is shown in [4] that prenex formulas of $\mathbf{G}$ allow for de-Skolemization. We generalize this result to proofs of prenex hypersequents. Our main tool is the following result from [11].

Theorem 2 (Mid-hypersequents). Any cut-free HG-proof $\sigma$ of a prenex hypersequent $\mathscr{H}$ can be stepwise transformed into one in which no propositional rule is applied below any application of a quantifier rule.

We call a hypersequent $\overline{\mathscr{H}}_{S}$ a linked Skolem instance of $\mathscr{H}$ if each formula $A$ in $\overline{\mathscr{H}}_{S}$ is an instance of a Skolemized formula $A^{S}$ that occurs in $\mathscr{H}^{S}$ on the same side (left or right) of a component as $A$. Moreover we link $A$ to $A^{S}$. As we will see in Section 7, we obtain (cut-free proofs of) linked Skolem instances from step 5 (and 6) of hyperCERES.

Theorem 3 (De-Skolemization). Given a cut-free HG-proof $\hat{\rho}$ of a linked Skolem instance $\overline{\mathscr{H}}_{S}$ of a prenex hypersequent $\mathscr{H}$, we can find a HG-proof $\rho$ of $\mathscr{H}$.

Proof. We construct $\rho$ in stages as follows:

1. By applying Theorem 2 to $\hat{\rho}$ we obtain a proof $\rho^{\prime}$ of the following form:

$$
\begin{array}{ccccc}
\rho_{1}^{p} & \cdots & \rho_{i}^{p} & \cdots & \rho_{n}^{p} \\
\mathscr{G}_{1} & & \mathscr{G}_{i} & & \mathscr{G}_{n} \\
& \ddots & \frac{\rho^{\mathrm{Q}}}{\mathscr{H}_{S}}
\end{array} .{ }
$$

where the mid-hypersequents $\mathscr{G}_{1}, \ldots, \mathscr{G}_{n}$ separate $\rho^{\prime}$ into a part $\rho^{\mathrm{Q}}$ containing only (weak) quantifier introductions and applications of structural rules and parts $\rho_{1}^{p}, \ldots, \rho_{n}^{p}$ containing only propositional and structural inferences.

2. Applications of the weakening rules, $(i w-l)$ and $(e w)$, can be shifted upwards to the axioms in the usual manner, while applications of $(i w-r)$ can be safely deleted by replacing each axiom $\perp \Rightarrow$ in the proof by $\perp \Rightarrow \Delta$ for suitable $\Delta$.

Consequently, $\rho^{\mathrm{Q}}$ does not contain weakenings after this transformation step.

3. Note that - in contrast to LK - Theorem 2 induces many and not just one midhypersequents, in general. The reason for this is the possible presence of the binary structural rule $(\mathrm{com})$ in $\rho^{\mathrm{Q}}$. To obtain a proof $\rho^{\prime \prime}$ with a single mid-hypersequent, we have to move 'communications' upwards in $\rho^{\mathrm{Q}}$; i.e., we have to permute applications of $(\mathrm{com})$ with applications of $(i c),(e c),(\forall-l)$, and $(\exists-r)$, respectively. The 
only non-trivial case is $(\forall-l)$. Disregarding side-hypersequents, the corresponding transformation consists in replacing

$$
\frac{\frac{\Gamma, P(x), \Sigma \Rightarrow \Delta}{\Gamma, \forall x P(x), \Sigma \Rightarrow \Delta}(\forall-l) \quad \Gamma, \forall x P(x), \Sigma \Rightarrow \Delta^{\prime}}{\Gamma, \forall x P(x) \Rightarrow \Delta \mid \Sigma \Rightarrow \Delta^{\prime}}(\mathrm{com})
$$

by

$$
\begin{aligned}
& \frac{\Gamma, P(x), \Sigma \Rightarrow \Delta}{\overline{\Gamma, P(x), \Sigma, \Gamma, \forall x P(x) \Rightarrow \Delta}}(i w)^{*} \quad \frac{\Gamma, \forall x P(x), \Sigma \Rightarrow \Delta^{\prime}}{\overline{\Gamma, P(x), \Sigma, \Gamma, \forall x P(x) \Rightarrow \Delta^{\prime}}}(i w)^{*} \\
& \Gamma, P(x), \Sigma \Rightarrow \Delta^{\prime} \mid \Gamma, \forall x P(x) \Rightarrow \Delta \quad \text { (com) } \Gamma, P(x), \Sigma \Rightarrow \Delta \\
& \frac{\frac{\Gamma, P(x) \Rightarrow \Delta\left|\Sigma \Rightarrow \Delta^{\prime}\right| \Gamma, \forall x P(x) \Rightarrow \Delta}{\Gamma, \forall x P(x) \Rightarrow \Delta\left|\Sigma \Rightarrow \Delta^{\prime}\right| \Gamma, \forall x P(x) \Rightarrow \Delta}(\forall-l)}{\Gamma, \forall x P(x) \Rightarrow \Delta \mid \Sigma \Rightarrow \Delta^{\prime}}(e c)
\end{aligned}
$$

4. For the final step we proceed like in [4], where the soundness of re-introducing strong quantifier occurrences for corresponding Skolem terms is shown: we ignore $\rho^{\prime \prime}$ and, given $\mathscr{H}$ and the links to its formulas, apply appropriate inferences to the mid-hypersequent as follows.

(a) Infer all weak quantifier occurrences, which can be introduced at this stage according to the quantifier prefixes in $\mathscr{H}$.

(b) Apply all possible internal and external contractions.

(c) Among the strong quantifiers that immediately precede the already introduced quantifiers we pick one linked to an instance of a Skolem term, that is maximal with respect to the subterm ordering. This term is replaced everywhere by the eigenvariable of the corresponding strong quantifier inference.

These three steps are iterated until the original hypersequent $\mathscr{H}$ is restored.

\section{Characteristic hyperclauses and reduced proofs}

All information of the original HG-proof $\sigma$ that goes into the cut-formulas is collected in a set $\Sigma_{d}(\hat{\sigma})$, consisting of hypersequents whose components only contain atomic formulas on the left hand sides and a (possibly empty) disjunction of atomic formulas, on the right hand side. We will call hypersequents of this latter form d-hyperclauses. In the proof of Theorem 4 we will construct characteristic d-hyperclauses $D_{i}$ together with corresponding reduced proofs $R_{i}(\hat{\sigma})$ which combine the cut-irrelevant part of the Skolemized proof $\hat{\sigma}$ with $D_{i}$. The pairs $\left\langle R_{i}(\hat{\sigma}), D_{i}\right\rangle$ provide the information needed to construct corresponding proofs containing only atomic cuts.

To assist concise argumentation we assume that the components of all hypersequents in a proof are labelled with unique sets of identifiers. More precisely, a derivation $\sigma$ is labelled if there is a function from all components of hypersequents occurring in $\sigma$ into the powerset of a set of identifiers, satisfying the following conditions: (We will put the label above the corresponding sequent arrow.) 
- All components occurring in initial hypersequents of $\sigma$ are assigned pairwise different singleton sets of identifiers.

- In all unary inferences the labels are transferred from the upper hypersequent to the lower hypersequent in the obvious way. In external weakening $(e w)$ a fresh singleton set is assigned to the new component in the lower hypersequent. In external contraction $(e c)$, if $\Gamma \stackrel{M}{\Rightarrow} \Delta$ and $\Gamma \stackrel{N}{\Rightarrow} \Delta$ are the two contracted components of the upper hypersequent, then $\Gamma^{M \cup N} \Delta$ is the corresponding component in the lower hypersequent.

- In all binary logical inferences the labels in the side-hypersequents are transferred in the obvious way, and the label of the component containing the main formula is the union of the labels of the components containing the active formulas.

- In (cut) the labels of the components containing the cut formulas are merged, like above, to obtain the label of the exhibited component of the lower hypersequent.

- In (com) the labels of all components are transferred from the premises to the lower hypersequent simply in the same sequence as exhibited in the statement of the rule.

Let $\mathscr{H}$ and $\mathscr{G}$ denote the labelled hypersequents

$$
\Gamma_{1} \stackrel{K_{1}}{\Rightarrow} \Delta_{1}|\cdots| \Gamma_{k} \stackrel{K_{k}}{\Rightarrow} \Delta_{k} \mid \mathscr{H}^{\prime} \quad \text { and } \quad \Gamma_{1}^{\prime} \stackrel{K_{1}}{\Rightarrow} \Delta_{1}^{\prime}|\cdots| \Gamma_{k}^{\prime} \stackrel{K_{k}}{\Rightarrow} \Delta_{k}^{\prime} \mid \mathscr{G}^{\prime}
$$

respectively, where the labels in $\mathscr{H}^{\prime}$ and $\mathscr{G}^{\prime}$ are pairwise different and also different from the labels $K_{1}, \ldots, K_{k}$. Then $\mathscr{H} \odot \mathscr{G}$ denotes the merged hypersequent

$$
\Gamma_{1}, \Gamma_{1}^{\prime} \stackrel{K_{1}}{\Rightarrow} \Delta_{1} \vee \Delta_{1}^{\prime}|\cdots| \Gamma_{k}, \Gamma_{k}^{\prime} \stackrel{K_{k}}{\Rightarrow} \Delta_{k} \vee \Delta_{k}^{\prime}\left|\mathscr{H}^{\prime}\right| \mathscr{G}^{\prime}
$$

where $\Delta_{i} \vee \Delta_{i}^{\prime}$ is $\Delta_{i}$ if $\Delta_{i}^{\prime}$ is empty and is $\Delta_{i}^{\prime}$ if $\Delta_{i}$ is empty (and thus $\Delta_{i} \vee \Delta_{i}^{\prime}$ is empty if both are empty).

Theorem 4. Given a Skolemized and labelled $\mathbf{H G}$-proof $\hat{\sigma}$ of $\mathscr{H}_{\hat{\sigma}}$ one can construct a characteristic set of pairs $\left\{\left\langle R_{1}(\hat{\sigma}), D_{1}\right\rangle, \ldots\left\langle R_{n}(\hat{\sigma}), D_{n}\right\rangle\right\}$, where, for all $i \in\{1, \ldots, n\}$, $D_{i}$ is a labelled d-hyperclause and $R_{i}(\hat{\sigma})$ is a labelled '(reduced)' cut-free $\mathbf{H G}$-proof with the following properties:

(1) the end-hypersequent of $R_{i}(\hat{\sigma})$ is $\mathscr{H}_{\hat{\sigma}}^{\prime} \odot D_{i}$, for some sub-hypersequent $\mathscr{H}_{\hat{\sigma}}^{\prime}$ of $\mathscr{H}_{\hat{\sigma}}$,

(2) the characteristic d-hyperclause set $\Sigma_{d}(\hat{\sigma})=\left\{D_{1}, \ldots, D_{n}\right\}$ is unsatisfiable.

Proof. To show (1) and (2) we use the following induction hypotheses:

(1') A characteristic set of pairs $\left\langle R_{i}\left(\hat{\sigma}^{\prime}\right), D_{i}^{\prime}\right\rangle$ exists for every sub-proof $\hat{\sigma}^{\prime}$ of $\hat{\sigma}$, where $R_{i}\left(\hat{\sigma}^{\prime}\right)$ proves $\mathscr{H}_{\hat{\sigma}^{\prime}}^{\prime} \odot D_{i}^{\prime}$ for some sub-hypersequent $\mathscr{H}_{\hat{\sigma}^{\prime}}^{\prime}$ of $\mathscr{H}_{\hat{\sigma}^{\prime}}$ which is cutirrelevant with respect to the original cuts in $\hat{\sigma}$. Moreover, the right hand sides in $\mathscr{H}_{\hat{\sigma}^{\prime}}^{\prime} \odot D_{i}^{\prime}$ are formulas in either $\mathscr{H}_{\hat{\sigma}^{\prime}}^{\prime}$ or in $D_{i}^{\prime}$.

(2') There is a derivation of the cut-relevant part of $\mathscr{H}_{\hat{\sigma}^{\prime}}$ from the set $\left\{D_{1}^{\prime}, \ldots, D_{m}^{\prime}\right\}$ of d-hyperclauses constructed for $\hat{\sigma}^{\prime}$.

Note that (2) follows from (2') as the cut-relevant part of $\mathscr{H}_{\hat{\sigma}}$ is an empty hypersequent by definition. The proof proceeds by induction on the length of $\hat{\sigma}^{\prime}$.

If $\hat{\sigma}^{\prime}$ consists just of an axiom $A \stackrel{M}{\Rightarrow} A$ then there is only one pair $\left\langle R\left(\hat{\sigma}^{\prime}\right), D\right\rangle$ in the corresponding characteristic set. $R\left(\hat{\sigma}^{\prime}\right)$ is the axiom itself and $D$ is the cut-relevant part 
of $A \stackrel{M}{\Rightarrow} A$ (which might be the empty hypersequent). (1') and (2') trivially hold. Axioms of the form $\perp \Rightarrow$ are handled in the same way.

If $\hat{\sigma}^{\prime}$ is not an axiom we distinguish cases according to the last inference in $\hat{\sigma}^{\prime}$. $(\vee-l): \hat{\sigma}^{\prime}$ ends with the inference

$$
\begin{array}{cc}
\vdots \hat{\rho} & \vdots \hat{\tau} \\
\frac{\mathscr{H} \mid A_{1}, \Gamma_{1} \stackrel{M}{\Rightarrow} \Delta}{\mathscr{H}\left|\mathscr{H}^{\prime}\right| A_{1} \vee A_{2}, \Gamma_{1}, \Gamma_{2} \stackrel{M \cup N}{\Rightarrow} \Delta} & \mathscr{H}^{\prime} \mid A_{2}, \Gamma_{2} \stackrel{N}{\Rightarrow} \Delta
\end{array}(\mathrm{V}-l)
$$

By induction hypothesis (1') there are characteristic sets of pairs $S_{1}=\left\{\left\langle R_{1}(\hat{\rho}), E_{1}\right\rangle, \ldots\right.$, $\left.\left\langle R_{m}(\hat{\rho}), E_{m}\right\rangle\right\}$ and $S_{2}=\left\{\left\langle R_{1}(\hat{\tau}), F_{1}\right\rangle, \ldots,\left\langle R_{n}(\hat{\tau}), F_{n}\right\rangle\right\}$, where the reduced proofs $R_{i}(\hat{\rho})$ and $R_{j}(\hat{\tau})$ end in $\mathscr{H}_{R_{i}(\hat{\rho})}=\mathscr{G}_{i} \odot E_{i}$ and in $\mathscr{H}_{R_{i}(\hat{\tau})}=\mathscr{G}_{j}^{\prime} \odot F_{j}$, respectively, where $\mathscr{G}_{i}$ and $\mathscr{G}_{j}^{\prime}$ are sub-hypersequents of the cut-irrelevant parts of $\mathscr{H} \mid A_{1}, \Gamma_{1} \stackrel{M}{\Rightarrow} \Delta$ and $\mathscr{H}^{\prime} \mid A_{2}, \Gamma_{2} \stackrel{N}{\Rightarrow} \Delta$, respectively. Moreover, by (2'), there are derivations $\rho_{C}$ and $\tau_{C}$ of the cut-relevant parts of the just mentioned hypersequents from $\left\{E_{1}, \ldots, E_{m}\right\}$ and $\left\{F_{1}, \ldots, F_{n}\right\}$, respectively.

Two cases can occur:

(a) If the inference is cut-relevant, then the characteristic set $S$ of pairs corresponding to $\hat{\sigma}^{\prime}$ is just $S_{1} \cup S_{2}$. Condition (1') trivially remains satisfied. Also (2') is maintained because we obtain a derivation of the cut-relevant part of $\mathscr{H}\left|\mathscr{H}^{\prime}\right|$ $A_{1} \vee A_{2}, \Gamma_{1}, \Gamma_{2} \stackrel{M \cup N}{\Rightarrow} \Delta$ by joining $\rho_{C}$ and $\tau_{C}$ with the indicated application of $(\vee-l)$.

(b) If the inference is cut-irrelevant, then we obtain the set $S$ corresponding to $\hat{\sigma}^{\prime}$ by

$$
S=\left\{\left\langle R_{i j}\left(\hat{\rho} \bowtie_{\vee-l} \hat{\tau}\right), E_{i} \bowtie_{i j} F_{j}\right\rangle: 1 \leq i \leq m, 1 \leq j \leq n\right\},
$$

where $R_{i j}\left(\hat{\rho} \bowtie_{\vee-l} \hat{\tau}\right)$ and $E_{i} \bowtie_{i j} F_{J}$ are defined as follows.

1. If $A_{1}$ does not occur at the indicated position in $\mathscr{H}_{R_{i}(\hat{\rho})}$ then $R_{i j}\left(\hat{\rho} \bowtie_{\vee-l} \hat{\tau}\right)$ is $R_{i}(\hat{\rho})$ and $E_{i} \bowtie_{i j} F_{j}$ is $E_{i}$.

2. If $A_{2}$ does not occur at the indicated position in $\mathscr{H}_{R_{j}(\hat{\tau})}$ then $R_{i j}\left(\hat{\rho} \bowtie_{\vee-l} \hat{\tau}\right)$ is $R_{j}(\hat{\tau})$ and $E_{i} \bowtie_{i j} F_{j}$ is $F_{j}$.

3. If neither $A_{1}$ nor $A_{2}$ occur as indicated in the reduced proofs, then $R_{i j}\left(\hat{\rho} \bowtie_{\vee-l} \hat{\tau}\right)$ can be non-deterministically chosen to be either $R_{i}(\hat{\rho})$ or $R_{j}(\hat{\tau})$ and $E_{i} \bowtie_{i j} F_{j}$ is either $E_{i}$ or $F_{j}$, accordingly.

4. If both $A_{1}$ and $A_{2}$ occur at the indicated positions, then $E_{i} \bowtie_{i j} F_{j}$ is $E_{i}^{\prime} \odot F_{j}^{\prime}$, where $E_{i}^{\prime}\left(F_{j}^{\prime}\right)$ is like $E_{i}\left(F_{j}\right)$, except for changing the label $M(N)$ to $M \cup N$.

Note that our labelling mechanism guarantees that the appropriate components are identified in merging hypersequents.

The corresponding reduced proof $R_{i j}\left(\hat{\rho} \bowtie_{\vee-l} \hat{\tau}\right)$ is constructed as follows. Since $A_{1}$ and $A_{2}$ occur as exhibited in the end-hypersequents $\mathscr{G}_{i} \odot E_{i}$ and $\mathscr{G}_{j}^{\prime} \odot F_{j}$ of $R_{i}(\hat{\rho})$ and $R_{j}(\hat{\tau})$, respectively, we want to join them by introducing $A_{1} \vee A_{2}$ using $(\vee-l)$ like in $\hat{\sigma}^{\prime}$. However, $(\vee-l)$ is only applicable if the right hand sides of the two relevant components in the premises are identical. To achieve this, we might first have to apply $(\vee-r)$ or $(i w-r)$ to the mentioned end-hypersequents. The resulting new end-hypersequent might still contain different components transferred from $E_{i}$ and $F_{j}$, respectively, that need to be merged with other components. This can be achieved by first applying internal weakenings to make the relevant components identical, and then applying external contraction $(e c)$ to remove redundant copies of identical components. 
Note that in all four cases (1') remains satisfied by definition of $R_{i j}\left(\hat{\rho} \bowtie_{\mathrm{V}-l} \hat{\tau}\right)$ and of $E_{i} \bowtie_{i j} F_{j}$. For cases 1, 2, and 3 also (2') trivially still holds. To obtain (2') for case 4 , we proceed in two steps. First we merge the occurrences of clauses $E_{1}, \ldots, E_{m}$ in the derivation $\rho_{C}$ of the cut-relevant part $\mathscr{H}_{c}^{\hat{\rho}}$ of $\mathscr{H}_{\hat{\rho}}$ with clauses in $\left\{F_{1}, \ldots, F_{n}\right\}$ to obtain a derivation $\rho_{C}\left(F_{i}\right)$ of $\mathscr{H}_{c}^{\hat{\rho}} \odot F_{i}$ for each $i \in\{1, \ldots, n\}$. In a second step, each initial hypersequent $F_{i}$ in the derivation $\tau_{C}$ of the cut-relevant part of $\mathscr{H}_{\hat{\imath}}$ is replaced by $\rho_{C}\left(F_{i}\right)$. By merging also the inner nodes of $\tau_{C}$ with $\mathscr{H}_{c}^{\hat{\rho}}$ we arrive at a derivation of the cut-relevant part of $\mathscr{H}_{\hat{\sigma}^{\prime}}$. (Actually, as the rules of HG are multiplicative, redundant copies of identical formulas might arise, that are to be removed by finally applying corresponding contractions.)

$\left(\wedge_{i}-l\right),(\supset-r),(\vee-r),(\forall-l),(\forall-r),(\exists-l),(\exists-r),(i c-l)$ : If the indicated last (unary) inference is cut-relevant, then the characteristic set of pairs remains the same as for the sub-proof ending with the premise of this inference.

If the inference is cut-irrelevant, then the hyperclauses $E_{1}, \ldots, E_{m}$ of the pairs in characteristic set $\left\{\left\langle R_{1}(\hat{\rho}), E_{1}\right\rangle, \ldots\left\langle R_{m}(\hat{\rho}), E_{m}\right\rangle\right\}$ for $\hat{\rho}$ remain unchanged. Each reduced proof $R_{i}(\hat{\rho})$ is augmented by the corresponding inference if its active formula occurs in the end-hypersequent $\mathscr{H}_{R_{i}(\hat{\rho})}$. If this is not the case then also $R_{i}(\hat{\rho})$ remains unchanged.

In any of these cases, $\left(1^{\prime}\right)$ and ( $\left.2^{\prime}\right)$ clearly remain satisfied.

$(e w),(i w-l),(i w-r)$ : The characteristic set of pairs remains unchanged and consequently (1') still holds. Also (2') trivially remains valid if the inference is cut-irrelevant. If a cut-relevant formula is introduced by weakening, then the derivation required for (2') is obtained from the induction hypothesis by adding a corresponding application of a weakening rule.

$(\wedge-r),(\supset-l),($ cut $),($ com $)$ : These cases are analogous to the one for $(\vee-l)$.

Example 1. Consider the labelled proof $\sigma$ in Figure 1.

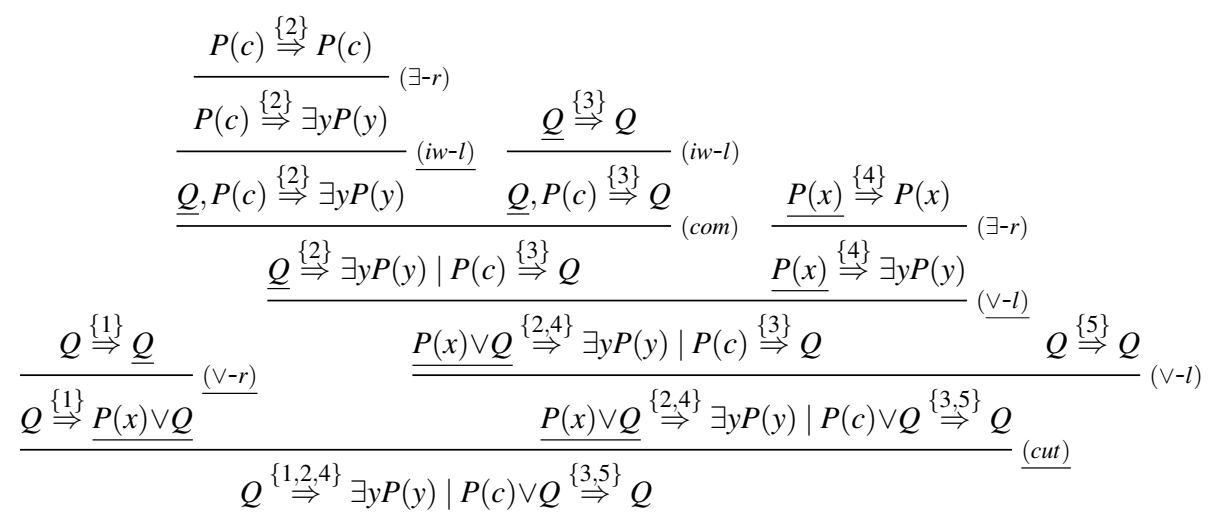

Fig. 1. Labelled proof $\sigma$ with underlined cut-relevant part.

The cut-relevant parts of $\sigma$ and the names of all corresponding cut-relevant infer-

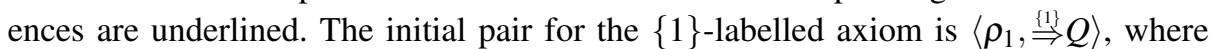


$\rho_{1}$ is $Q \stackrel{\{l\}}{\Rightarrow} Q$. Since the succeeding inference $(\vee-r)$ is unary and cut-relevant, the pair remains unchanged in that step.

For the middle part of the proof let us look at the subproof $\sigma^{\prime}$ ending with an application of $(\mathrm{com})$ yielding $Q \stackrel{\{2\}}{\Rightarrow} \exists y P(y) \mid P(c) \stackrel{\{⿰ 3\}}{\Rightarrow} Q$. Since there are no cut-ancestors in the $\{2\}$-labelled axiom, the corresponding d-hyperclause is the empty $\stackrel{\{2\}}{\Rightarrow}$. This is retained for the right premise of $(\mathrm{com})$. The corresponding reduced derivation consists only of the first inference $(\exists-r)$ as the succeeding application of $(i w-l)$ is cut-relevant. For the left premise of the communication we obtain the d-hyperclause $Q \stackrel{\{3\}}{\Rightarrow}$, which is then merged and 'communicated' with $\stackrel{\{2\}}{\Rightarrow}$ to obtain for $\sigma^{\prime}$ the d-hyperclause $Q \stackrel{\{2\}}{\Rightarrow} \mid \stackrel{\{3\}}{\Rightarrow}$. This forms a pair with the reduced derivation $R\left(\sigma^{\prime}\right)$, which, in this case, is identical with $\sigma^{\prime} .^{7}$

From the cut-relevant (and therefore underlined) $(\vee-l)$-inference one obtains an additional pair $\left\langle\rho_{2}, P(x) \stackrel{\{4\}}{\Rightarrow}\right\rangle$ from its right premise, where $\rho_{2}$ is the derivation of $P(x) \stackrel{[4\}}{\Rightarrow} \exists y P(y)$ from the axiom.

For the succeeding cut-irrelevant application of $(\mathrm{V}-l)$, the pair $\left\langle\rho_{2}, P(x) \stackrel{\{4\}}{\Rightarrow}\right\rangle$ remains unchanged, as the left disjunct $P(x)$ does not occur at the left side in the endhypersequent $\stackrel{[4\}}{\Rightarrow} \exists y P(y)$ of $\rho_{2}{ }^{8}$ The reduced proof $\rho_{3}$ of the final pair is formed by applying $(\vee-l)$ as indicated to the end-hypersequent of $R\left(\sigma^{\prime}\right)$ and to $Q \stackrel{\{f\}}{\Rightarrow} Q$ as right and left premises, respectively. The corresponding d-hyperclause arises from merging $Q \stackrel{\{2\}}{\Rightarrow} \mid \stackrel{\{3\}}{\Rightarrow}$ and $\stackrel{\{5\}}{\Rightarrow}$ into $Q \stackrel{\{2\}}{\Rightarrow} \mid \stackrel{\{3,5\}}{\Rightarrow}$.

For the final application of cut we have to take the union of the sets of pairs constructed for its two premises. Therefore the characteristic set of pairs for $\sigma$ is

$$
\left\{\left\langle\rho_{1}, \stackrel{\{1\}}{\Rightarrow} Q\right\rangle,\left\langle\rho_{2}, P(x) \stackrel{\{4\}}{\Rightarrow}\right\rangle,\left\langle\rho_{3}, Q \stackrel{\{2\}}{\Rightarrow} \mid \stackrel{\{3,5\}}{\Rightarrow}\right\rangle\right\} .
$$

It is easy to check that conditions (1) and (2) of Theorem 4 are satisfied.

\section{Hyperclause resolution}

By a hyperclause we mean a hypersequent in which only atomic formulas occur. Remember that, from the proof of Theorem 4, we obtain d-hyperclauses, which are like hyperclauses, except for allowing disjunctions of atomic formulas at the right hand sides of their components. However, using the derivable rule (distr) (see Section 2) it is easy to see that an HG-derivation of, e.g., the d-hyperclause

$$
A \Rightarrow B \vee C \mid \Rightarrow D \vee E \vee F
$$

can be replaced by an HG-derivation of the hyperclause

$$
A \Rightarrow B|A \Rightarrow C| \Rightarrow D|\Rightarrow E| \Rightarrow F .
$$

\footnotetext{
${ }^{7}$ Note that neither the cut-relevant application of $(i w-l)$ nor $Q$ appears in the reduced proof corresponding to $Q, P(c) \stackrel{\{2\}}{\Rightarrow} \exists y P(y)$. Still, the missing $Q$ is added by $(i w-l)$ in $R\left(\sigma^{\prime}\right)$ to make the application of $(\mathrm{com})$ possible.

8 This is case $(\vee-l) /(b) / 2$ in the proof of Theorem 4.
} 
Also the converse holds: using the rules $\left(\vee_{i}-r\right)$, and $(e c)$ we can derive the mentioned dhyperclause from the latter hyperclause. Therefore we can refer to hyperclauses instead of d-hyperclauses in the following.

We also want to get rid of occurrences of $\perp$ in hyperclauses. Since $\perp \Rightarrow$ is an axiom, any hyperclause which contains an occurrence of $\perp$ at the left hand side of some component is valid. But such hyperclauses are redundant, as our aim is to construct refutations for unsatisfiable sets of hyperclauses. On the other hand, any occurrence of $\perp$ at the right hand side of a component is also redundant and can be deleted. In other words: we can assume without loss of generality that $\perp$ does not occur in hyperclauses. (Note that this does not imply that occurrences of $\perp$ are removed from HG-proofs.)

In direct analogy to classical resolution, the combination of a cut-inference and most general unification is called a resolution step. The lower hyperclause in

$$
\frac{\mathscr{H}\left|\Gamma_{1} \Rightarrow A \quad \mathscr{H}^{\prime}\right| A^{\prime}, \Gamma_{2} \Rightarrow \Delta}{\theta\left(\mathscr{H}\left|\mathscr{H}^{\prime}\right| \Gamma_{1}, \Gamma_{2} \Rightarrow \Delta\right)} \text { (res) }
$$

where $\theta$ is the most general unifier of the atoms $A$ and $A^{\prime}$, is called resolvent of the premises, that have to be variable disjoint. If no variables occur, and thus $\theta$ is empty, (res) turns into (cut) and we speak of ground resolution. The soundness of this inference step is obvious. We show that hyperclause resolution is also refutationally complete. It is convenient to view hyperclauses as sets of atomic sequents. This is equivalent to requiring that external contraction is applied whenever possible. Consequently, there is a unique unsatisfiable hyperclause, namely the empty hyperclause. A derivation of the empty hyperclause by resolution from initial hypersequents contained in a set $\Sigma$ of hyperclauses is called a resolution refutation of $\Sigma$.

As usual for resolution, we focus on inferences on ground hyperclauses and later transfer completeness to the general level using a corresponding lifting lemma.

Theorem 5. For every unsatisfiable set of ground hyperclauses $\Psi$ there is a ground resolution refutation of $\Psi$.

Proof. We proceed by induction on $e(\Psi)=\|\Psi\|-|\Psi|$, where $\|\Psi\|$ is the total number of occurrences of atoms in $\Psi$, and $|\Psi|$ is the cardinality of $\Psi$.

If $e(\Psi) \leq 0$ then either $\Psi$ already contains the empty hyperclause, or else $\Psi$ contains exactly one atom per hyperclause. In the latter case, as $\Psi$ is unsatisfiable, there must be hyperclauses $C_{1}=(\Rightarrow A)$ and $C_{2}=(A \Rightarrow)$ in $\Psi$. Obviously the empty clause is a ground resolvent of $C_{1}$ and $C_{2}$.

$e(\Psi) \geq 1: \Psi$ must contain a hyperclause $C$ that has more than one atom occurrence. Without loss of generality let $C=(\mathscr{H} \mid \Gamma \Rightarrow A)$, where $\Gamma$ may be empty. (The case where all atoms in $C$ occur only on the left hand side of sequents is analogous.) Since $\Psi$ is unsatisfiable also the sets $\Psi^{\prime}=(\Psi-\{C\}) \cup\{\mathscr{H} \mid \Gamma \Rightarrow\}$ and $\Psi^{\prime \prime}=(\Psi-\{C\}) \cup\{\Rightarrow A\}$ must be unsatisfiable. Since $e\left(\Psi^{\prime}\right)<e(\Psi)$ and $e\left(\Psi^{\prime \prime}\right)<e(\Psi)$ we obtain ground resolution refutations $\rho^{\prime}$ of $\Psi^{\prime}$ and $\rho^{\prime \prime}$ of $\Psi^{\prime \prime}$, respectively. By adding in $\rho^{\prime}$ an occurrence of $A$ to the right side of the derived empty hyperclause and likewise to all other hyperclauses in $\rho^{\prime}$ that are on a branch ending in the initial hyperclause $\mathscr{H} \mid \Gamma \Rightarrow$, we obtain a resolution derivation $\rho_{A}^{\prime}$ of $\Rightarrow A$ from $\Psi$. By replacing each occurrence of $\Rightarrow A$ as initial hyperclauses in $\rho^{\prime \prime}$ by a copy of $\rho_{A}^{\prime}$ we obtain the required ground resolution refutation of $\Psi$. 
Remark. Note that our completeness proof does not use any special properties of $\mathbf{G}$. Only the polarity between left and right hand side of sequent and the disjunctive interpretation of '|' at the meta-level are used. For any logic $\mathscr{L}$ : whenever we can reduce $\mathscr{L}$-validity (or $\mathscr{L}$-unsatisfiability) of a formula $F$ to $\mathscr{L}$-unsatisfiability of a corresponding set of atomic hyperclauses, we may use hyperclause resolution to solve the problem.

To lift Theorem 5 to general hyperclauses, one needs to add (the hypersequent version of) factorization:

$$
\frac{\mathscr{H} \mid \Gamma \Rightarrow \Delta}{\theta\left(\mathscr{H} \mid \Gamma^{\prime} \Rightarrow \Delta\right)} \text { (factor) }
$$

where $\theta$ is the most general unifier (see, e.g., [15]) of some atoms in $\Gamma$ and where $\theta \Gamma^{\prime}(\theta)$ is $\theta(\Gamma)$ after removal of copies of unified atoms. The lower hyperclause is called a factor of the upper one.

Lemma 1. Let $C_{1}^{\prime}$ and $C_{2}^{\prime}$ be ground instances of the variable disjoint hyperclauses $C_{1}$ and $C_{2}$, respectively. For every ground resolvent $C^{\prime}$ of $C_{1}^{\prime}$ and $C_{2}^{\prime}$ there is a resolvent $C$ of factors of $C_{1}$ and $C_{2}$.

The proof of Lemma 1 is exactly as for classical resolution (see, e.g., [15]) and thus is omitted here. Combining Theorem 5 and Lemma 1 we obtain the refutational completeness of general resolution.

Corollary 1. For every unsatisfiable set of hyperclauses $\Sigma$ there is a resolution refutation of $\Sigma$.

We will make use of the observation that any general resolution refutation of $\Sigma$ can be instantiated into (essentially) a ground resolution refutation of a set $\Sigma^{\prime}$ of instances of hyperclauses in $\Sigma$, whereby resolution steps turn into cuts and factorization turns into additional contraction steps. (Note that additional contractions do not essentially change the structure of a ground resolution refutation.)

\section{Projection of hyperclauses into HG-proofs}

Remember that from Theorem 4 (in Section 5) we obtain a characteristic set of pairs $\left\{\left\langle R_{1}(\hat{\sigma}), D_{1}\right\rangle, \ldots\left\langle R_{n}(\hat{\sigma}), D_{n}\right\rangle\right\}$ for the proof $\hat{\sigma}$ of $\mathscr{H}^{S}$. As described in Section 6, we can construct a resolution refutation $\gamma$ of the hyperclause set $\left\{C_{1}, \ldots, C_{n}\right\}$ corresponding to the d-hyperclauses $\left\{D_{1}, \ldots, D_{n}\right\}$. (This is step 3 of hyperCERES.) Forming a ground instantiation $\gamma^{\prime}$ of $\gamma$ yields a derivation of the empty hypersequent that consists only of atomic cuts and contractions. (Step 4 of hyperCERES.) Each leaf node of $\gamma^{\prime}$ is a ground instance $\theta\left(C_{i}\right)$ of a hyperclause in $\left\{C_{1}, \ldots, C_{n}\right\}$. From Theorem 4 we also obtain, for each $i \in\{1, \ldots, n\}$ a cut-free proof $R_{i}(\hat{\sigma})$ of $\mathscr{G}_{i} \odot D_{i}$, where $\mathscr{G}_{i}$ is a sub-hypersequent of the cut-irrelevant part of $\mathscr{H}_{\hat{\sigma}}$ and $D_{i}$ is the d-hyperclause corresponding to $C_{i}$. We instantiate $R_{i}(\hat{\sigma})$ using $\theta$ and finally apply (distr), as indicated in Section 6, to obtain a cut-free proof $\hat{\sigma}_{i}^{\theta}$ of $\theta\left(\mathscr{G}_{i}\right) \odot \theta\left(C_{i}\right)$.

To get a proof $\gamma^{\prime}[\hat{\sigma}]$ of a linked Skolem instance of the original hypersequent $\mathscr{H}$ (cf. Section 4) we replace each leaf node $\theta\left(C_{i}\right)$ of $\gamma^{\prime}$ with the proof $\hat{\sigma}_{i}^{\theta}$ of $\theta\left(\mathscr{G}_{i}\right) \odot \theta\left(C_{i}\right)$, 
described above, and transfer the instances $\theta\left(\mathscr{G}_{i}\right)$ of cut-irrelevant formulas in $\mathscr{H}$ also to the inner nodes of $\gamma^{\prime}$ in the obvious way, i.e., to regain correct applications of atomic cuts. As mentioned in Section 3, the remaining atomic cuts can easily be removed from $\gamma^{\prime}[\hat{\sigma}]$. The resulting proof is subjected to de-Skolemization as described in Theorem 3. This final step 7 of hyperCERES yields the desired cut-free proof of $\mathscr{H}$.

Example 2. We continue Example 1, where we have obtained the characteristic set of pairs $\left\{\left\langle\rho_{1}, \stackrel{\{1\}}{\Rightarrow} Q\right\rangle,\left\langle\rho_{2}, P(x) \stackrel{\{4\}}{\Rightarrow}\right\rangle,\left\langle\rho_{3}, Q \stackrel{\{2\}}{\Rightarrow} \mid \stackrel{\{3,5\}}{\Rightarrow}\right\rangle\right\}$ for the proof $\sigma$ of the (trivially) Skolemized prenex hypersequent $Q^{\{1,2,4\}} \exists y P(y) \mid P(c) \vee Q \stackrel{\{3,5\}}{\Rightarrow} Q$.

The obtained d-hyperclauses are in fact already hyperclauses. Moreover, one can immediately see that the hyperclauses $\stackrel{\{1\}}{\Rightarrow} Q$ and $Q \stackrel{\{2\}}{\Rightarrow} \mid \stackrel{\{3,5\}}{\Rightarrow}$ can be refuted by a one-step resolution derivation $\gamma$ :

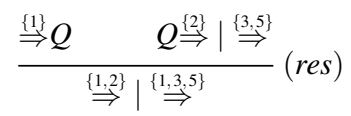

Note that $P(x) \stackrel{\{4\}}{\Rightarrow}$ and the corresponding reduced proof $\rho_{2}$ are redundant. In our case, $\gamma$ is already ground. Therefore no substitution has to be applied to the reduced proofs $\rho_{1}$ and $\rho_{3}$. By replacing the two upper (d-)hyperclauses in $\gamma$ with $\rho_{1}$ and $\rho_{3}$, respectively we obtain the desired proof $\gamma[\sigma]$ that only contains an atomic cut:

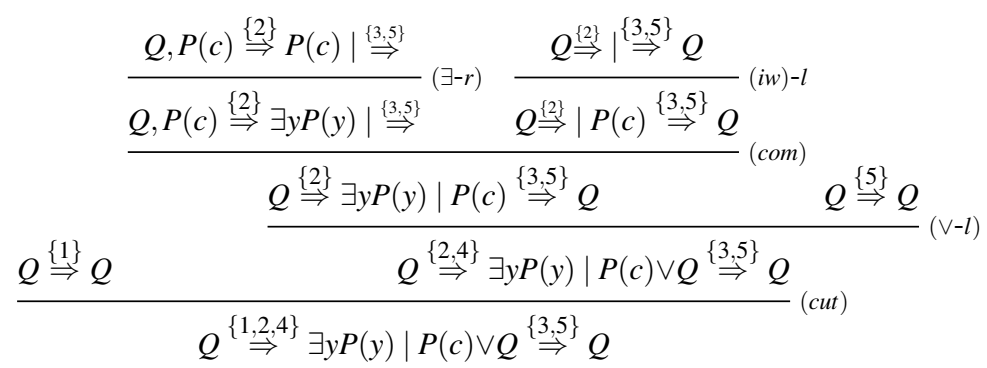

\section{Final remarks}

The results of this paper are easily extendable to larger fragments G: (de-)Skolemization is sound already for intuitionistic logic I without positive occurrences of universal quantifiers, if an additional existence predicate is added [6]. Therefore hyperCERES applies after incooperation of the mentioned existence predicate. Other classes where Skolemization is sound for $\mathbf{I}$ are described by Mints [16].

The most interesting question however is whether hyperCERES can be extended to intuitionistic logic itself. Note that we obtain a calculus for I by dropping the communication rule from HG. It turns out that hyperCERES is applicable to the class of (intuitionistic) hypersequents not containing negative occurrences of $\vee$ or positive occurrences of $\forall$, as the distribution rule (distr) is still sound for this fragment of $\mathbf{I}$. This fragment actually is an extension of the Harrop class [14] with weak quantifiers.

The extendability of hyperCERES to full intuitionistic logic depends on the development of an adequate (de-)Skolemization technique, together with a concept of parallelized resolution refutations, that takes into account the disjunctions of atoms at the right hand side of clauses without using (distr). 
From a more methodological viewpoint, it should be mentioned that hyperCERES uses the fact that 'negative information' can be treated classically in intermediate logics like $\mathbf{G}$, and that cuts amount to entirely negative information in our approach. In this sense, global cut elimination, as presented in this paper, is more adequate for intermediate logics than stepwise reductions, which treat cuts as positive information.

\section{References}

1. A. Avron: Hypersequents, Logical Consequence and Intermediate Logics for Concurrency. Annals of Mathematics and Artificial Intelligence 4, 225-248 (1991)

2. A. Avron: The Method of Hypersequents in Proof Theory of Propositional Non-Classical Logics. In Logic: From Foundations to Applications. Clarendon Press, 1-32 (1996)

3. M. Baaz and A. Ciabattoni: A Schütte-Tait style cut-elimination proof for first-order Gödel logic. Proceedings of Tableaux 2002, LNAI 2381, 24-38 (2002)

4. M. Baaz, A. Ciabattoni, C.G. Fermüller: Herbrand's Theorem for Prenex Gödel Logic and its Consequences for Theorem Proving. Proc. of LPAR'2001. LNAI 2250, 201-216 (2001)

5. M. Baaz, S. Hetzl, A. Leitsch, C. Richter, and H. Spohr: CERES: An Analysis of Fürstenberg's Proof of the Infinity of Primes, to appear in Theoretical Computer Science

6. M. Baaz, R. Iemhoff: The Skolemization of existential quantifiers in intuitionistic logic. Ann. of Pure and Applied Logics 142 269-295 (2006)

7. M. Baaz, A. Leitsch: Cut-elimination and Redundancy-elimination by Resolution. J. Symb. Comput. 29(2), 149-177 (2000)

8. M. Baaz, A. Leitsch: CERES in Many-Valued Logics. Proceedings of LPAR'2005, LNAI 3452, 1-20 (2005)

9. M. Baaz, A. Leitsch: Towards a clausal analysis of cut-elimination. J. Symb. Comput. 41(34), 381-410 (2006)

10. M. Baaz, A. Leitsch, R. Zach: Incompleteness of an infinite-valued first-order Gödel Logic and of some temporal logic of programs. Proc. of CSL'95, LNCS 1092, 1-15 (1996)

11. M. Baaz, R. Zach: Hypersequents and the Proof Theory of Intuitionistic Fuzzy Logic. Proc. CSL'2000, 187-201 (2000)

12. A. Chagrov, M. Zakharyaschev: Modal Logic. Oxford University Press (1997)

13. P. Hájek: Metamathematics of Fuzzy Logic. Kluwer (1998)

14. R. Harrop: Concerning formulas of the types $A \supset B \vee C, A \supset(\exists x) B(x)$ in intuitionistic formal systems J. Symbolic Logic 25, 27-32 (1960)

15. A. Leitsch: The Resolution Calculus. Springer (1997)

16. G. Mints: The Skolem method in intuitionistic calculi. Proc. Inst. Steklov 121, 73-109 (1974)

17. V. P. Orevkov: Lower Bounds for Increasing Complexity of Derivations after Cut Elimination. J. Soviet Mathematics, 2337-2350 (1982)

18. K. Schütte: Beweistheorie. Springer, (1960)

19. R. Statman: Lower bounds on Herbrand's theorem. Proc. of the Amer. Math. Soc. 75, 104 107 (1979)

20. W.W. Tait: Normal derivability in classical logic. In The Syntax and Semantics of infinitary Languages, LNM 72, 204-236 (1968)

21. A.S. Troelstra, H. Schwichtenberg: Basic Proof Theory. Second edition, Cambridge (2000)

22. G. Takeuti, T. Titani: Intuitionistic fuzzy logic and intuitionistic fuzzy set theory. J. Symbolic Logic, 49, 851-866 (1984) 